Dorin N. Poenaru is Professor at the National Institute of Physics and Nuclear Engineering, Bucharest and is frequently invited to the Frankfurt Institute for Advanced Studies. He is mentioned in Encyclopaedia Britannica with A. Sandulescu and W. Greiner for predicting heavy particle radioactivity.

Both authors have fulfilled terms as scientific directors of their institute in Bucharest.

\section{References}

[1] G.A. Proca, Alexandre Proca. Oeuvre Scientifique Publiée, S.I.A.G., Rome, 1988.

[2] A. Calboreanu, Rom. J. Phys. 49 (2004) 3.

[3] http://www.th.physik.uni-frankfurt.de/ poenaru/PROCA/Proca.html (2005). D.N. Poenaru, E-print, physics/0508195, http://arXiv.org, 2005.

[4] W. Pauli, Nobel lecture, December 13, 1946.

[5] W. Pauli, Rev. Mod. Phys. 13 (1941) 213.

[6] C. P. Enz, No time to be brief (A scientific biography of Wolfgang Pauli), Oxford University Press, New York, 2004.
[8] D. Levrett, A.S. Goldhaber, M.M. Nieto, Phys. Rev. Lett. 35 (1975) 1402. A.S. Goldhaber, M.M. Nieto, Scientific American 234 (1976) 86.

[9] C. Vuille, J. Ipser, J. Gallagher, “Einstein-Proca model, micro black holes, and naked singularities", General Relativity and Gravitation, 34 (2002) 689.

[10] R. Tomaschitz, Physica A 293 (2001) 247; A 335 (2004) 577; Eur. Phys. J. D 32 (2005) 241.

[11] W. Greiner, Relativistic quantum mechanics; W. Greiner and J. Reinhardt, Field quantization; W. Greiner and A. Schäfer, Quantum chromodynamics, Springer, Berlin, 2000, 1996, 1995, respectively.

[12] R. Scipioni, "Isomorphism between non-Riemannian gravity and Einstein-Proca-Weyl theories extended to a class of scalar gravity theories", Class. Quantum Grav., 16 (1999) 2471.

[13] R. W. Tucker and C. Wang, C., "An Einstein-Proca-fluid model for dark matter gravitational interactions", Nucl. Phys. B - Proc. suppl., 57 (1997) 259.

[14] H. Belich, T. Costa-Soares, M. M. Ferreira Jr., J. A. Helay-Neto, "Classical solutions in a Lorentz violating scenario of MaxwellChern-Simons-Proca electrodynamics”, Eur. Phys. J. C 42 (2005) 127.

[7] L.-C. Tu, J. Luo, G. T. Gillies, Rep. Prog. Phys. 68 (2005) 77.

\title{
Water from Heaven
}

\author{
L.J.F. (Jo) Hermans, \\ Leiden University•The Netherlands•E-mail: Hermans@Physics.LeidenUniv.nl
}

$\mathrm{L}$ arge rain drops fall faster than small ones, that much is obvious for any physicist. But let's be a bit more precise. The terminal velocity follows from the balance between the weight of the drop and its air resistance. What exactly is the resistance of a drop falling through the atmosphere? We have to distinguish two regimes here. If the droplets are very small, like cloud droplets (or fog particles, if you wish), the Reynolds number is so small that Stokes' formula applies: the air resistance is proportional to viscosity, radius and velocity: $F=6 \pi \eta R v$. For a typical cloud droplet having a radius of $0.01 \mathrm{~mm}$, we find a terminal velocity of about $1 \mathrm{~cm} / \mathrm{s}$. That is very small indeed. But it goes up rapidly with size: since its weight is proportional to $\mathrm{R}^{3}$ and the resistance only to $\mathrm{R}$, the terminal velocity increases with the square of the size for such droplets. That applies to droplets up to about $0.1 \mathrm{~mm}$ in diameter, according to the handbooks (good old Ludwig Prandtl's book Führung durch die Strömungslehre, for example).

For ordinary raindrops, above about $1 \mathrm{~mm}$ diameter, turbulent flow dominates. Here the weight is balanced by drag $F_{D}=C_{D} \pi R^{2} \cdot 1 / 2 \rho v^{2}$, where $\pi R^{2}$ is the frontal area,

$C_{D}$ is the drag coefficient, which is about 0.5

for a sphere at the relevant Reynolds numbers, and $\rho$ the density of air. For a rain drop of $1 \mathrm{~mm}$ in diameter, we find a terminal velocity of $16 \mathrm{~km} / \mathrm{h}$. Note that in this regime the velocity is proportional to the square root of the diameter. Consequently, a $3 \mathrm{~mm}$ raindrop reaches $28 \mathrm{~km} / \mathrm{h}$. And so on, we would guess. Given the above, we may expect that for the biggest drops - $5 \mathrm{~mm}$, say - the terminal velocity is well above $35 \mathrm{~km} / \mathrm{h}$.

Wrong! Something interesting happens, as already noticed by the German physicist, Philipp Lenard, a century ago. Using a vertical wind tunnel to balance the speed of the drop, he noticed that drops larger than about $3 \mathrm{~mm}$ diameter become deformed to the shape of a small pancake, and have a flat bottom. Consequently, their frontal area is larger than for spherical droplets having the same mass. As a result of the increased drag, the terminal velocity hardly increases any further: for raindrops of 4 and $5 \mathrm{~mm}$ it reaches an asymptotic value of about $29 \mathrm{~km} / \mathrm{h}$, which is practically the same speed as that already reached by the $3 \mathrm{~mm}$ drops.

And beyond $5 \mathrm{~mm}$ ? As soon as the diameter reaches about $5.5 \mathrm{~mm}$, the forces become so large that surface tension cannot hold the drop together, so it breaks up into pieces. For raindrops, there is no life beyond $5 \mathrm{~mm}$ !

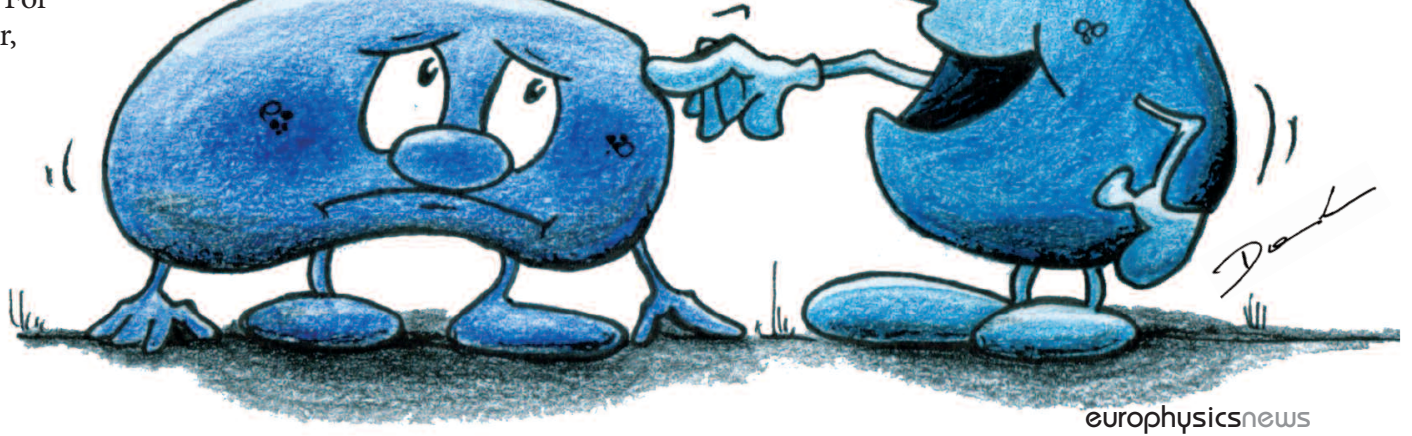

